\title{
Antibiotic stewardship programs and the internist's role
}

\author{
Massimo Giusti, Elena Cerutti \\ S.C. di Medicina per Intensità di Cure, Ospedale San Giovanni Bosco, ASL TORINO 2, Italy
}

\begin{abstract}
Antimicrobial resistance (AMR) is a worldwide issue, but with significant epidemiological diversity in different countries. A recently observed phenomenon is represented by the diffusion of AMR, initially confined to intensive-care units, to medical wards. This was predictable, since patients hospitalized in medical units are made up of more than 70\% of elderly people (over 75 years of age in 1 case out of 2). They are fragile patients, with significant comorbidity (over a half with at least 3 diseases), weakened immune systems, and consequently a higher risk of infection. Given such a scenario, it becomes therefore both necessary and urgent to adopt a multifaceted approach of antimicrobial stewardship programs in order to prevent, detect and control the emergence of antimicrobial resistant organisms. The ideal antimicrobial program is led by an infectious diseases (ID) physician and clinical pharmacist with ID training, together with a list of other important staff: clinical microbiologist, information systems specialist, infection control professional, and hospital epidemiologist. In real life, not all the Italian hospitals have got an ID physician and therefore the best canditates for antimicrobial management practices are internists if provided with a specific expertise in ID and antibiotic therapy. Adhering to the principles of optimal antimicrobial therapy in their clinical practice, the internist is able to improve the care and help to reduce the resistance of a patient at his bedside. At the same time, he can achieve other key goals reducing the length of stay and reducing the cost and utilization of health care resources.
\end{abstract}

\section{Introduction}

In 1895, Vincenzo Tiberio, a medical officer of the Medical Corps in the Italian Navy, described the bactericidal properties of some molds and, in 1928, Alexander Fleming succeeded in isolating penicillin; so giving rise to what would later become antibiotics. In 1941, the first experiment was carried out on humans and, two years later, the use of antibiotics was authorized in a military hospital.

From that moment onwards, the use of antibiotics in clinics revolutionized the approach to the treatment of infections that had hitherto been thought incurable.

However, Sir Alexander Fleming himself, during his speech at the Nobel Prize ceremony in 1945, warned

Correspondence: Massimo Giusti, S.C. di Medicina per Intensità di Cure, Ospedale San Giovanni Bosco, ASL TORINO 2, p.zza del Donatore di Sangue 3, 10154 Torino, Italy.

E-mail: massimogiusti@libero.it

Key words: Antibiotics; antimicrobial resistance; sepsis.

Received for publication: 6 October 2016.

Accepted for publication: 20 October 2016.

This work is licensed under a Creative Commons Attribution NonCommercial 4.0 License (CC BY-NC 4.0).

(C) Copyright M. Giusti and E. Cerutti, 2016

Licensee PAGEPress, Italy

Italian Journal of Medicine 2016; 10:329-338

doi:10.4081/itjm.2016.797 that it was necessary to use the new drugs carefully if they were to be used effectively; and this, because the microbes were capable of learning how to protect themselves and developing strategies for their survival. $\mathrm{He}$ introduced the concept that antimicrobial resistance (AMR) is the expression of the evolutionary adaptation of the bacteria, which takes place concomitantly with the introduction of the antimicrobials into therapy.

Antibiotics are the only drug where the use in one patient can impact the effectiveness in another.

There exists, in fact, a strong connection between the use of antibiotics and the appearance of AMR.

The ecological pressure produced by antibiotics on bacteria provokes a situation of extreme Darwinian advantage for the microorganisms that are able to resist: the sensitive ones are destroyed, while those resistant emerge.

The main mechanisms of AMR emerge by mutation and selection, or by means of horizontal transference, supported by plasmids (genetic exchange), of genes capable of giving resistance to the main classes of antibiotics by way of multifaceted mechanisms: outflowing pumps, alteration of the membrane pores, modification of the sites of action, or enzymatic inactivation of the drug.

In the early days of antibiotics, booming drug development meant that even when resistance developed, a new drug was always available to treat the increasingly resistant bacteria. Fourteen new classes of antibiotics were introduced between 1935 and 2003.

Unfortunately, for at least two decades now, the search for new antibiotics has been proven less profitable than that to develop drugs to treat chronic dis- 
ease, and consequently our capacity to effectively cure infective diseases has been seriously impaired. Today, the lack of an effective therapy is cause for great concern worldwide, and it could take us right back to the pre-antibiotic era.

The effects of AMR are present in all the main bacterial pathogens responsible for infections in medical wards patients, but the greatest clinical impact is caused by the methicillin-resistant Staphylococcus aureus (MRSA), by vancomycin-resistant Enterococcus (VRE), by Enterobacteriaceae producers of extendedspectrum $\beta$-lactamase (ESBL) and, more recently, also carbapenemase, and multiresistant and non-fermenting gramnegative bacteria, such as Pseudomonas aeruginosa and Acinetobacter baumannii.

The increase in mortality, morbidity, and prolonged hospitalization, as well as added costs incurred by the use of antibiotics and more expensive procedures, constitute the main clinical implications of germ-resistant infections.

Bacteremia $^{1,2}$ and surgical site infections ${ }^{3}$ due to MRSA have been associated with a higher mortality rate than similar infections due to methicillin-susceptible $S$. aureus, with the mean attributable cost of an MRSA infection ranging from $\$ 9275$ to $\$ 13,901 .{ }^{4}$ Similarly, compared with vancomycin-susceptible Enterococcus faecium infections, bloodstream infections due to VRE were associated with decreased survival ( $24 \%$ vs $59 \%)$, increased length of hospital stay (34.8 vs 16.7 days), and an attributable cost of $\$ 27,190$ per episode., 5 A metaanalysis of 9 studies of VRE bloodstream infections found an attributable excess mortality of $30 \%$, compared with vancomycin-susceptible Enterococcus bloodstream infections. ${ }^{7}$ Similar adverse outcomes have also been reported for infections with resistant gramnegative organisms, including Pseudomonas, Acinetobacter, and Enterobacter species and ESBL-producing organisms. ${ }^{8}$ A case-control study found that third-generation cephalosporin-resistant Enterobacter infections were associated with increased mortality (relative risk, $5.02)$, length of hospital stay (1.5-fold increase), and an attributable cost of $\$ 29,379 .{ }^{9}$

Analyses from the European Centre for Disease Prevention and Control (ECDC) in 2009 estimated that infections caused by a subset of resistant bacteria are responsible for about 25,000 deaths in Europe annually.

In addition to these avoidable deaths, healthcare costs and productivity losses have been estimated to be at least EUR 1.5 billion. $^{10}$

The major drivers behind the occurrence and spread of AMR are the use of antimicrobial agents, not only for human health, and the transmission of antimicrobial-resistant microorganisms between humans, between animals, and between humans, animals and the environment. While antimicrobial use exerts ecological pressure on bacteria and contributes to the emergence and selection of AMR, poor infection prevention and control practices and inadequate sanitary conditions favor the further spread of these bacteria.

For the first time, the ECDC, the European Food Safety Authority (EFSA) and the European Medicines Agency (EMA) have jointly explored associations between consumption of antimicrobials in humans and food-producing animals, and antimicrobial resistance in bacteria from humans and food-producing animals, using 2011 and 2012 data currently available from their relevant five EU monitoring networks.

The consumption of several antimicrobials extensively used in animal husbandry was higher in animals than in humans, while consumption of antimicrobials critically important for human medicine (such as fluoroquinolones and $3^{\text {rd }}$ - and $4^{\text {th }}$-generation cephalosporins) was higher in humans. In both humans and animals, positive associations between consumption of antimicrobials and the corresponding resistance in bacteria were observed for most of the combinations investigated. In some cases, a positive association was also found between antimicrobial consumption in animals and resistance in bacteria from humans. ${ }^{11}$

\section{Antimicrobial resistance in Italy and in internal medicine wards}

AMR is a worldwide problem, but with diverse epidemiological significance in different countries.

In Italy, the phenomenon is particularly evident for most bacterial pathogens. The presence of MRSA is twice that of the European average. It is, in fact, just under $40 \%$ (38\% in 2014) compared with the $20 \%$ European average. Of importance is also the proportion of macrolide-resistant pneumococci (Italy, with $27 \%$, has the highest number in Europe), while the proportion of penicillin-insensitive pneumococci has been lower in the last decade, around $10 \%$. Among the gram-negative pathogens, Escherichia coli e Klebsiella pneumoniae resistant to fluoroquinolones and cephalosporins, of the third generation, have spread rapidly in our country, reaching very high proportions $(41 \%$ and $46 \%$, respectively, for the resistance to fluoroquinolones; $20 \%$ and $46 \%$, respectively, for the resistance to third-generation cephalosporins). This phenomenon has caused a considerable increase in the administration of carbapenems, which has, in turn, brought about the diffusion of carbapenem-resistant Acinetobacter and Enterobacter. ${ }^{12}$

This exceptional concentration of resistance in Italy can be explained, on the one hand, by the scarce attention paid to prevention measures; on the other hand, by the abuse or bad use of antibiotics.

A recently observed phenomenon is represented by the diffusion of AMR, initially confined to intensive-care units, to medical wards.

Today, there is a $20 \%$ infection incidence in Internal Medicine wards similar to what has been observed 
in Intensive Therapy Units (14-24\%) and Hematology-Oncology ones.

This was predictable, since patients hospitalized in medical units are made up of more than $70 \%$ of elderly people (over 75 years of age in 1 case out of 2). They are fragile patients, with significant comorbidity (over a half with at least 3 diseases), weakened immune systems, and consequently a higher risk of infection.

In internal medicine patients, bacterial infections cause $30 \%$ of mortality, and constitute one of the most common causes of hospital treatment.

Apart from old age, other conditions exist in the internal medicine wards that constitute the main risks of infection by difficult germs: central venous catheters and urinary catheters, mechanical ventilation, the ample use of antibiotics, the recent hospitalization in welfare structures, the total parenteral nutrition, hemodialysis and chemotherapy.

Therefore, it is easily understood why the AMR percentages of $S$. aureus, E. coli and K. pneumonia in Internal Medicine today are much higher than the national average.

Another factor that worsens the situation is the frequent resort to antibiotics in internal medicine patients. For example, the computer physician order entry (CPOE) for the prescription and administering of drugs in the internal medical department of San Giovanni Bosco hospital, in Turin (Prisma-Software), shows that the prevalence in the usage of antibiotics in our ward [1800 admissions to hospital/year, by Emergency Department (ED)] has progressively increased from $40 \%$ to $50 \%$ from 2004 to 2015 . The adoption of alternatives to normal hospitalization (Day Hospital, Day Service, etc.) accessible by the ED for many pathologies (cardiac failure, venous thromboembolism, chronic obstructive pulmonary disease, oncological pathology, etc.), has brought about a relative increase in hospitalization for acute or complicated conditions that cannot be handled in any other way; like fever syndromes and infections (respiratory, urinary, abdominal, circulatory, cutaneous, tissue, amongst others), consequently leading to a greater use of antibiotics. These, in fact, constitute one of the main, and in some cases the main, cost factor of the pharmacy in an Internal Medicine ward. To this must be added that resorting to antibiotic therapy is not always justified.

It would appear that $30 \%$ of antibiotics prescribed in hospitals in the USA are without effect and inappropriate. Therefore, it is necessary and of top priority to improve the use of antibiotics, above all in hospitals, for the safety of patients and public health in general. ${ }^{13,14}$

\section{What is antimicrobial stewardship}

In recognition that antimicrobial resistance results in increased morbidity, mortality, and cost of health care, the Infectious Diseases Society of America (IDSA) initially published guidelines for improving the use of antimicrobial agents in hospitals in $1988^{15}$ and then jointly published guidelines with the Society for Healthcare Epidemiology of America in 1997 for the prevention of antimicrobial resistance in hospitals. ${ }^{16}$

However, subsequent surveys of hospitals have found that practices to improve antimicrobial use are frequently inadequate and not implemented on a daily basis. ${ }^{17,18}$

In the face of such a scenario, it is necessary to adopt a multifaceted approach to prevent, detect, and control the emergence of antimicrobial-resistant organisms.

This includes ensuring the availability of adequate and appropriate therapeutic agents, the existence of diagnostic capacity to rapidly and reliably detect specific pathogens and their antimicrobial susceptibilities, and the promotion of robust infection prevention, control, and antimicrobial stewardship programs (ASP).

ASP refers to coordinated interventions designed to improve and measure the appropriate use of antimicrobial agents by promoting the selection of the optimal antimicrobial drug regimen, including dosing, duration of therapy, and route of administration, that results in the best clinical outcome for the treatment or prevention of infection, with minimal toxicity to the patient and minimal impact on subsequent resistance. ${ }^{19}$

The goal of ASP is 3 fold.

The first goal is to work with health care practitioners to help each patient receiving the most appropriate antimicrobial with the correct dose and duration. Joseph and Rodvold wrote about the 4 D's of optima antimicrobial therapy: right $d \mathrm{rug}$, right $d \mathrm{ose}$, $d \mathrm{e}$-escalation to pathogen-directed therapy, and right $d$ uration of therapy. ${ }^{20}$ The optimal care of an infected patient means treating with the correct, properly dosed antibiotic and one that has the least likelihood of causing collateral damage (i.e., leading to resistance in the patient or his or her contacts). An added benefit of programs that aim to optimize antibiotic use is that they generally experience cost savings because fewer doses of antibiotic are used and less expensive antibiotics are chosen. Comprehensive programs have demonstrated annual savings of $\$ 200,000$ to $\$ 900,000 .^{21,22}$

The second goal is to prevent antimicrobial overuse, misuse, and abuse. In both the hospital and the outpatient setting, physicians use antibiotics when they are not necessary. Antibiotics are given to patients with viral infections, noninfectious processes (a classic example is the febrile patient with pancreatitis), bacterial infections that do not require antibiotics (such as small skin abscesses that will resolve with incision and drainage), and bacterial colonization (as in the case of a positive urine culture result in a patient with a bladder catheter). 
Antibiotics are also frequently misused, such as in the very common scenario of the use of broad-spectrum antibiotics that cover multidrug resistant organisms in a patient whose infection was acquired in the community or the failure to adjust antibiotics according to culture data, thus maintaining the patient on a regimen to which the organism is not susceptible. Abuse of antibiotics is more difficult to define, but the term might be used to describe the use of one particular antibiotic preferentially over others by a physician as a result of aggressive detailing by the pharmaceutical representative or worse because of financial interest.

The third goal is to minimize the development of resistance. Both at individual patient level and at community level, antibiotic use changes susceptibility patterns. Patients exposed to antibiotics are at higher risk of becoming colonized or infected by resistant organisms. The most common cause of the development of Clostridium difficile diarrhea is exposure to antibiotics. Gram-negative resistance to carbapenems and cephalosporins has been shown to increase 10 to 20 fold with exposure to these broadspectrum antimicrobials.

In a recent systematic review and metaanalyses of outpatient prescribing practices, the use of common antibiotics was associated with significant increased risk of development of antibiotic resistance, up to 12 months after antimicrobial exposure [pooled odds ratio (OR), 1.33; 95\% confidence interval (CI), 1.21.5]. More importantly, antimicrobial resistance is associated with increased morbidity and mortality. Carbapenem resistant K. pneumoniae is associated with an increased attributable mortality compared with sensitive Klebsiella (OR, 4.69; 95\% CI, 1911.58; $\mathrm{P}=0.001)$ and methicillin-resistant $S$ aureus bacteremia, relative to methicillin-sensitive $S$. aureus bacteremia, has a significantly greater mortality risk as well (OR, 1.93; 95\% CI, 1542.42; $\mathrm{P}=0.001)$.

These resistant organisms can be transmitted to other individuals within the hospital or in the patient's community. Antimicrobial resistance also has significant hospital and societal costs. A recent study by Roberts et al. estimated that the cost of an antimicrobial resistant infection is $\$ 18,588$ to $\$ 29,069$ per patient, with an excess duration of hospital stay of 6.4 to 12.7 days and attributable mortality of $6.5 \%$.

\section{Stewardship team}

Every hospital should work according to the resources available to create a multidisciplinary interprofessional antimicrobial management team that is physician directed or supervised.

At a minimum, 1 or more members of the team should have training in ASP. The number of team members may vary on the basis of the size and complexity of the facility. Team members should include but are not limited to: i) a physician; ii) a pharmacist; iii) a clinical microbiologist; iv) an infection preventionist.

The 2007 IDSA/Society for Healthcare Epidemiology of America (SHEA) guidelines for ASPs defined the ideal antimicrobial program as led by an ID physician and clinical pharmacist with ID training, together with a list of other important staff: clinical microbiologist, information systems specialist, infection control professional, and hospital epidemiologist. Clearly, this is an extensive team requiring significant resources. Although optimal, many institutions do not have an ID physician on staff or an attending who is interested and willing to participate. Looking at the numbers, there simply are not enough ID physicians to fill this need. Many institutions will be unable to recruit an ID pharmacist with sufficient skill to manage an ASP. As a consequence, many institutions wanting to develop an ASP to improve clinical outcomes, reduce antimicrobial resistance, and lower costs will need to think outside the box and look for progressive leaders to champion and lead their programs. Potential nontraditional leaders include general clinical pharmacists, intensivists, hospitalists and internists. Furthermore, recognized leaders in the field of antibiotic stewardship have strongly supported the role of hospitalists and internists in the leadership of ASPs.

Unique to hospital medicine, and incorporated into the definition of the profession, are the commitment to quality and process improvement, efficient use of hospital and healthcare resources, and an interdisciplinary approach to care. ${ }^{23}$

Internists must address the metrics that matter most to the hospitals in which they work: length of stay excess days, patient satisfaction, readmissions, and resource utilization, to name a few.

Internists with an interest in infectious disease can be ideal physician leaders for efforts to improve antibiotic use given their increasing presence in inpatient care, the frequency with which they use antibiotics and their commitment to quality improvement. ${ }^{24,25}$

At the forefront of inpatient care, internists are well positioned as champions of the principles and practices of ASP. Internists can participate in ASP at a number of levels. On the direct patient care level, internists can consistently apply the principles of appropriate empiric therapy, de-escalation, and duration of therapy. By adhering to the principles of optimal antimicrobial therapy in their clinical practice, internists can improve care and help reduce resistance on a patient-by-patient basis. At the same time, they may achieve other key internist goals by reducing length of stay and decreasing costs and health care resource utilization. Collaboration between ID specialists and internists in ASP is a vast and mostly untapped resource. With the majority of 
US hospitals having both on staff, the potential is tremendous. Internists who do ASP work need the support of the local ID community for protocol development as well as a resource when clinical scenarios become complicated. On a wider scale, the active support and engagement of the respective professional societies [Federation of Associations of Hospital Doctors on Internal Medicine (FADOI) and Italian Society of Tropical and Infectious Diseases (SIMIT)] could stimulate significant expansion of ASPs across the country.

\section{Administrative support}

It is essential the support and collaboration between the antimicrobial stewardship team and the hospital infection control and pharmacy and therapeutics committees or their equivalents. The support and collaboration of hospital administration, medical staff leadership, and local providers in the development and maintenance of antimicrobial stewardship programs is essential to success of the program. In this regard, the infectious diseases physician and the head of pharmacy, as appropriate, should negotiate with hospital administration to obtain adequate authority, compensation, and expected outcomes for the program. It is essential the presence of hospital administrative support for the necessary infrastructure, to measure antimicrobial use and to track use on an ongoing basis. It is desirable that antimicrobial stewardship programs function under the auspices of quality assurance and patient safety. Prior to program implementation, the antimicrobial stewardship strategic plan should be presented to and approved by the chiefs of professional services, hospital medical staff executive committee, and/or other medical staff governing bodies, to ensure their acceptance and support.

\section{Stewardship strategies}

There is no single template for a program to optimize antibiotic prescribing in hospitals. The complexity of medical decision-making surrounding antibiotic use and the variability in the size and types of care among the hospitals require flexibility in implementation. However, experience demonstrates that antibiotic stewardship programs can be implemented effectively in a wide variety of hospitals and that success is dependent on defined leadership and a coordinated multidisciplinary approach. ${ }^{26,27}$

There are 2 major approaches to antimicrobial stewardship, with the most successful programs generally implementing a combination of both. The frontend or pre-prescription approach to stewardship uses restrictive prescriptive authority (Table 1). Certain antimicrobials are considered restricted and require prior authorization for use by all except a select group of clinicians. Clinicians without authority to prescribe the drug in question must contact the designated antimicrobial steward and obtain approval to order the antimicrobial. The frontend approach has the advantage of targeting specific antimicrobials for specific indications based on local resistance patterns and the hospital formulary. Antimicrobials can be approved for a specific duration, thereby prompting review after culture data have been obtained. Data suggest that programs that use this approach have been able to demonstrate significant reductions in expenditures of the targeted drug but also result in increased use of antimicrobials that are not restricted, which may or may not be the desired effect.

This intervention requires the availability of expertise in antibiotic use and infectious diseases and authorization needs to be completed in a timely manner.

The backend or post-prescription approach to stewardship uses prospective review and feedback (Table 2). The antimicrobial steward reviews current antibiotic orders and provides clinicians with recommendations to continue, adjust, change, or discontinue the therapy based on the available microbiology results and clinical features of the case. Studies of programs that use this approach have shown decreased antimicrobial use, decreased number of new prescriptions of antimicrobials, and improved clinician satisfaction. The backend approach has the advantage of being able to focus on de-escalation, a critical aspect of appropriate antimicrobial use. De-escalation is modification of the initial empiric antimicrobial regimen based on culture data, other laboratory tests, and the clinical status of the patient.

De-escalation includes changing a broad-spectrum antibiotic to one with narrower coverage, changing from combination therapy to monotherapy, or stopping antibiotic therapy altogether as it becomes more apparent that these drugs are not needed.

Audit and feedback requires the availability of expertise and some smaller facilities have shown success by engaging external experts to advise on case reviews.

\section{Table 1. Restrictive interventions.}

- Limitation of antibiotics available in the hospital list

- Motivated and nominal request for individual patient for specific antibiotics

- Expert opinion (Infection diseases specialist) for use of certain classes of antibiotics

- Limitation of prescription for certain classes of antibiotics only by some specialists 


\section{Restrictive versus persuasive interventions}

The metaanalysis of 52 ITS studies was used to compare restrictive versus purely persuasive interventions. Restrictive interventions had significantly greater impact on prescribing outcomes at one month ( $32 \%, 95 \%$ CI $2 \%$ to $61 \%, \mathrm{P}=0.03)$ and on microbial outcomes at 6 months $(53 \%, 95 \%$ CI $31 \%$ to $75 \%$, $\mathrm{P}=0.001$ ) but there were no significant differences at 12 or 24 months. Interventions intended to decrease excessive prescribing were associated with reduction in $C$. difficile infections and colonization or infection with aminoglycosideor cephalosporin-resistant gramnegative bacteria, MRSA and VRE. Metaanalysis of clinical outcomes showed that four interventions intended to increase effective prescribing for pneumonia were associated with significant reduction in mortality (risk ratio $0.89,95 \% \mathrm{CI} 0.82$ to 0.97 ), whereas nine interventions intended to decrease excessive prescribing were not associated with significant increase in mortality (risk ratio $0.92,95 \%$ CI 0.81 to 1.06 ). ${ }^{28}$

\section{Supplemental antimicrobial stewardship strategies}

\section{Education}

Education is considered to be an essential element of any program designed to influence prescribing behavior and can provide a foundation of knowledge that will enhance and increase the acceptance of stewardship strategies.

However, education alone, without incorporation of active intervention, is only marginally effective in changing antimicrobial prescribing practices and has not demonstrated a sustained impact.

\section{Guidelines and clinical pathways}

Clinical practice guidelines are being produced with increasing frequency, with the goal of ensuring highquality care. However, the impact on provider behavior and improved clinical outcomes has been difficult to measure. Although physicians usually agree, in principle, with national guidelines, the absence of accompanying strategies for local implementation often presents a formidable barrier. Antimicrobial stewardship programs can facilitate multidisciplinary development of

Table 2. Persuasive interventions.

\footnotetext{
- Educational meetings about guidelines in antibiotic therapy

- Periodic meeetings with prescribers

- Educational materials in printed or informatics form for the prescribers
}

evidence-based practice guidelines that incorporate local microbiology and resistance patterns.

\section{Antimicrobial cycling}

There are insufficient data to recommend the routine use of antimicrobial cycling as means of preventing or reducing antimicrobial resistance over a prolonged period of time. Substituting one antimicrobial for another may transiently decrease selection pressure and reduce resistance to the restricted agent. Unless the resistance determinant has been eliminated from the bacterial population, however, reintroduction of the original antimicrobial is again likely to select for the expression of the resistance determinant in the exposed bacterial population.

\section{Combination therapy: prevention of resistance versus redundant antimicrobial coverage}

The rationale for combination antimicrobial therapy includes broad-spectrum empirical therapy for serious infections, improved clinical outcomes, and the prevention of resistance.

However, in many situations, combination therapy is redundant and unnecessary.

There are insufficient data to recommend the routine use of combination therapy to prevent the emergence of resistance. Combination therapy does have a role in certain clinical contexts, including use for empirical therapy for critically ill patients at risk of infection with multidrug-resistant pathogens, to increase the breadth of coverage and the likelihood of adequate initial therapy.

\section{Streamlining or de-escalation of therapy}

Streamlining or de-escalation of empirical antimicrobial therapy on the basis of culture results and elimination of redundant combination therapy can more effectively target the causative pathogen, resulting in decreased antimicrobial exposure and substantial cost savings.

\section{Dose optimization}

Optimization of antimicrobial dosing that accounts for individual patient characteristics (e.g., age, renal function, and weight), causative organism and site of infection (e.g., endocarditis, meningitis, and osteomyelitis), and pharmacokinetic and pharmacodynamic characteristics of the drug is an important part of antimicrobial stewardship. Examples in practice include prolonged or continuous infusion of $\beta$-lactams, extended-interval dosing of aminoglycosides, and dosing of fluoroquinolones for Streptococcus pneumoniae in community-acquired pneumonia and for Pseudomonas in hospital-acquired pneumonia and ventilator-associated pneumonia. The use of 
pharmacokinetic and pharmacodynamics principles is more likely to be in development of antimicrobial use guidelines than in individual patients' care.

Optimization of antimicrobial dosing based on individual patient characteristics, causative organism, site of infection, and pharmacokinetic and pharmacodynamic characteristics of the drug is an important part of antimicrobial stewardship.

\section{Intravenous-to-oral switch therapy}

Antimicrobial therapy for patients with serious infections requiring hospitalization is generally initiated with parenteral therapy. Enhanced oral bioavailability among certain antimicrobials, such as fluoroquinolones, oxazolidinones, metronidazole, clindamycin, trimethoprim-sulfamethoxazole, fluconazole, and voriconazole, allows conversion to oral therapy once a patient meets defined clinical criteria.

A systematic plan for parenteral to oral conversion of antimicrobials with excellent bioavailability, when the patient's condition allows, can decrease length of hospital stay, health care costs and potential complications due to intravenous access. Development of clinical criteria and guidelines allowing conversion to use of oral agents can facilitate implementation at the institutional level.

\section{Rapid microbiological diagnosis}

The role of diagnostic laboratory testing is another area of evolution. Rapid diagnostic tests such as procalcitonin, fluorescence in situ hybridization using peptide nucleic acid probes, and matrix-assisted laser desorption/ionization time of flight (MALDI-TOF) mass spectrometric analysis have been successfully incorporated by some stewardship programs and may become important additions to stewardship efforts..$^{29-32}$

Matrix-assisted laser desorption/ionization time-offlight mass spectrometry can rapidly (within $60 \mathrm{~min}$ ) identify bacteria, including rare species not ordinarily associated with clinical infection or pathogens that are difficult to grow or to identify to the species level.

The use of rapid molecular assays and mass spectrometry to identify bacterial species and susceptibility in blood cultures has been associated with statistically significant improvements in time to initiation of appropriate antibiotic therapy, rates of recurrent infection, mortality, length of stay, and hospital costs.

Some studies emphasize the importance of combining use of rapid testing with 2 strategies to maximize the benefits and likelihood of a favorable impact on outcomes. First, ASP support or rapid notification of results was a consistent feature of the studies that found statistically significant associations between rapid testing and outcomes. In contrast, studies lacking these features often did not find evidence of associa- tions between rapid testing and improved antibiotic use, time to initiation of appropriate antibiotic therapy, or length of stay benefit, despite shortening the time to pathogen identification. Second, rapid testing should be performed continuously (i.e., 24/7) or at least in frequent batches. The optimal implementation of rapid testing requires increased laboratory resources and additional costs.

\section{Computer surveillance and decision support}

Increased focus on medical errors and patient safety led to a series of reports by the Institute of Medicine's National Roundtable on Health Care Quality to emphasize the role of information technology in the delivery of health care.

The Leapfrog Group has identified CPOE, a form of patient management software, as 1 of the 3 most important leaps that organizations can take to substantially improve patient safety.

CPOE has the potential to incorporate clinical decision support and to facilitate quality monitoring.

The entered orders are communicated over a computer network to the medical staff or to the departments (pharmacy, laboratory, or radiology) responsible for fulfilling the order. CPOE decreases delay in: i) order distribution; ii) resource allocation; iii) order completion; and shall: i) reduce errors related to handwriting or transcription; ii) allow order entry at the point of care or off-site; iii) provide error-checking for duplicate or incorrect doses or tests; and iv) simplify inventory and posting of charges.

Progress to this end, however, remains slow, with only $<10 \%$ of Italian hospitals converting to electronic medical records.

Health care information technology in the form of electronic medical records, CPOE, and clinical decision support can improve antimicrobial decisions through the incorporation of data on patient-specific microbiology cultures and susceptibilities, hepatic and renal function, drug-drug interactions, allergies, and cost. However, implementation of these features has been slow, and conformation of the technology to the clinical environment remains a challenge.

Computer-based surveillance can facilitate good stewardship by more efficient targeting of antimicrobial interventions, tracking of antimicrobial resistance patterns, and identification of nosocomial infections and adverse drug events.

\section{How to evaluate the effectiveness of an antimicrobial stewardship program}

Although many healthcare institutions in the United States have implemented ASPs, the impact of these programs has been difficult to gauge. Currently, 
there is no consensus on optimal metrics to demonstrate the effectiveness of an ASP; thus, assessment of the true effect of these programs has been challenging. Metrics for ASPs can be divided into 4 main categories: patient outcomes, unintended consequences, antimicrobial utilization and costs, and process measures. Outcome metrics are of greatest interest because they reflect all aspects of care, and optimization of outcomes is the ultimate objective of the intervention. Because of the challenges surrounding the measurement of outcomes, process measures have often been used to demonstrate the impact of ASPs. Neither of these metrics alone is sufficient to describe the overall impact of stewardship efforts, and, to be most effective, assessments should include both patient-specific outcomes and related process measures to adequately assess the impact of any intervention. For example, studies that report only a reduction in antimicrobial use infer that the ASP has improved outcomes. Inferential data may not suffice to gain continued support for ASP efforts. Conversely, assessing patient outcome data without some quantification of associated stewardship strategies is not useful. In 2011, the IDSA Emerging Infections Network surveyed its membership to determine characteristics of ASPs and reported that clinicians and administrators differ in their assessments of outcomes of importance needed to support these programs. An overwhelming majority (83\%) of administrators underscored the importance of evidence of cost savings, whereas $63 \%-72 \%$ of physicians were more focused on patient outcomes, citing reductions in $C$. difficile infection, adverse events, and resistance rates as the most important indicators to justify an ASP. In another survey, ID physicians and pharmacists ranked appropriateness of antimicrobial use, infection-related mortality, and antimicrobial-associated length of stay as the metrics of highest importance to demonstrate the impact of an ASP. ${ }^{33}$

\section{Metrics to abandon}

For reasons described in the preceding section, we advocate abandoning drug costs as a metric of process or outcome for an ASP.

Antimicrobial acquisition costs do not reflect any of the primary goals of antimicrobial stewardship. Estimating the overall cost of care would provide a better measure of the savings accrued or costs avoided. Efforts should shift to measuring the value of care, with value defined as health outcomes achieved per dollar spent and cost referring to the total costs of care for the patient's medical condition, not the cost of individual services. It may be beneficial for ASP personnel to partner with the institution's finance department to help with determination of overall cost of care. As a primary strategy, routine individual drug audits or reviews should be replaced by evidence-based disease state reviews. A case may be made for targeting certain agents, for example, if there is considerable variability in use from year to year or if use of an agent is associated with a surge in resistance or adverse events. Otherwise, we consider review of use by disease state to be the preferable option.

Another metric that could be abandoned is the number of stewardship interventions performed or antimicrobials tracked, as these metrics provide no information on actual outcomes. What matters from a quality or safety perspective is the outcome of the protocol or therapy that was implemented as a result of the intervention. A couple of analogies can serve to illustrate this point. A fall-prevention strategy used in hospitals consisted of placing a sticker in the charts of at-risk patients; the metric tracked was not the number of stickers placed in patient charts but rather the number of falls that occurred. Similarly, standard isolation precautions are used in infection control, and the metric that is tracked is not the number of patients placed in isolation, but the actual infection rates. Correspondingly, with ASPs, the goal is to optimize antimicrobial therapy, not the number of times the pharmacist had to intervene. If monitoring interventions is necessary, possibly as means of tracking time spent on antimicrobial stewardship activities, data gathering should be facilitated by a robust IT infrastructure to limit busywork of ASP members. Each program should include appropriate audits of compliance, but not as a metric to demonstrate the impact or success of the program.

\section{Conclusions}

AMR is increasing in the departments of Internal Medicine all over the world. However, antimicrobial drug development is slowing.

Now more than ever before, it is of utmost importance to use properly antimicrobials to prevent the development of resistance and improve patient outcomes.

Current ASP guidelines ${ }^{34}$ outline the requirements and strategies for implementing ASPs but do not provide detailed input regarding the optimal metrics to gauge the success of ASPs. ${ }^{35}$

In many institutions, the focus is on antimicrobial costs and utilization, with patient outcomes reported less frequently.

The first targets of an ASP should be improving the quality of care for patients and minimizing antimicrobial resistance in society. ${ }^{36}$

A universal antimicrobial stewardship model does not exist. Each and every project must be tailored to its objective and the needs of the health care organization concerned; with implementation aims and modalities matching the inappropriateness of a given 
situation, and always bearing in mind the intervention needed and the staff required accomplishing it.

Various actions may be taken to reach preset goals in such projects, but of vital importance is the creation of work-teams of a multidisciplinary nature, in which infectious disease physicians, pharmacists, microbiologists and internists all collaborate with one another in an equally responsible manner in order to ensure effectiveness and stability in their work, and without which they would not be able to reach their common objectives.

In Italy, there are 12,000 professional internists in all the hospitals. They manage 39 thousand beds for 1.2 million admissions to hospital. They are highly specialized physicians who, thanks to a great expertise in nearly all medical subjects, globally take care of patients and not only of their pathologies, in an olistic way.

In many Italian hospitals, the specialists in Internal Medicine are promoters of appropriateness in health care, of quality improvement and of clinical governance; showing both management and economical capacities, but without in any way neglecting their patients' safety. The success of any multidisciplinary activity of antibiotic appropriateness always begins at the patient's bedside and this undoubtdly represents the internist's strength. ${ }^{37,38}$

Internists are ideally suited to be actively involved to promote ASPs at their institutions. By supporting the effort for hospitalized patients to receive appropriate antibiotic coverage, ASPs can decrease hospital lengths of stay, costs, and antimicrobial resistance, thereby aligning perfectly with the goals of many divisions of internal medicine.

\section{References}

1. Cosgrove SE, Sakoulas F, Perencevich EN, et al. Comparison of mortality associated with methicillin-resistant and methicillin-susceptible Staphylococcus aureus bacteremia: a meta-analysis. Clin Infect Dis 2003;36:53-9.

2. Melzer M, Eykyn SJ, Gransden WR, Chinn S. Is methicillin-resistant Staphylococcus aureus more virulent than methicillin-susceptible S. aureus? A comparative cohort study of British patients with nosocomial infection and bacteremia. Clin Infect Dis 2003;37:1453-60.

3. Engemann JJ, Carmeli Y, Cosgrove SE, et al. Adverse clinical and economic outcomes attributable to methicillin resistance among patients with Staphylococcus aureus surgical site infection. Clin Infect Dis 2003;36:592-8.

4. Chaix C, Durand-Zaleski I, Ablerti C, Brun-Buisson C. Control of endemic methicillin-resistant Staphylococcus aureus: a cost-benefit analysis in an intensive care unit. JAMA 1999;282:1745-51.

5. Stosor V, Peterson LR, Postelnick M, Noskin GA. Enterococcus faecium bacteremia: does vancomycin-resistance make a difference? Arch Intern Med 1998;158:522-7.

6. Diaz Granados CA, Zimmer SM, Klein M, Jernigan JA. Comparison of mortality associated with vancomycin- resistant and vancomycin-susceptible enterococcal bloodstream infections: a meta-analysis. Clin Infect Dis 2005;41:327-33.

7. Salgado CD, Farr BM. Outcomes associated with vancomycin-resistant enterococci: a meta-analysis. Infect Control Hosp Epidemiol 2003;24:690-8.

8. Cosgrove SE. The relationship between antimicrobial resistance and patient outcomes: mortality, length of hospital stay, and health care costs. Clin Infect Dis 2006;42: S82-9.

9. Cosgrove SE, Kaye KS, Eliopoulous GM, Carmeli Y. Health and economic outcomes of the emergence of third-generation cephalosporin resistance in Enterobacter species. Arch Intern Med 2002;162:185-90.

10. European Centre for Disease Prevention and Control. The bacterial challenge: time to react. Stockholm: ECDC; 2009. Available from: http://www.ecdc.europa.eu/en/publications/Publications/0909_TER_The_Bacterial_Challenge_Time_to_React.pdf

11. ECDC (European Centre for Disease Prevention and Control), EFSA (European Food Safety Authority) and EMA (European Medicines Agency). ECDC/EFSA/EMA first joint report on the integrated analysis of the consumption of antimicrobial agents and occurrence of antimicrobial resistance in bacteria from humans and foodproducing animals. Stockholm/Parma/London: ECDC/ EFSA/EMA; 2015. EFSA J 2015;13:4006.

12. European Centre for Disease Prevention and Control (ECDC). Antimicrobial resistance surveillance in Europe 2014. Annual Report of the European Antimicrobial Resistance Surveillance Network (EARS-Net). Stockholm: ECDC; 2015.

13. Camins BC, King MD, Wells JB, et al. Impact of an antimicrobial utilization program on antimicrobial use at a large teaching hospital: a randomized controlled trial. Infect Control Hosp Epidemiol 2009;30:931-8.

14. Fridkin SK, Baggs J, Fagan R, et al. Vital signs: improving antibiotic use among hospitalized patients. Morbid Mortal Wkly Rep 2014;63:194-200.

15. Marr JJ, Moffet HL, Kunin CM. Guidelines for improving the use of antimicrobial agents in hospitals: a statement by the Infectious Diseases Society of America. J Infect Dis 1988;157:869-76.

16. Shlaes DM, Gerding DN, John JF, et al. Society for Healthcare Epidemiology of America and Infectious Diseases Society of America joint committee on the prevention of antimicrobial resistance: guidelines for the prevention of antimicrobial resistance in hospitals. Clin Infect Dis 1997;25:584-99.

17. Lawton RM, Fridkin SK, Gaynes RP, McGowan JE. Practices to improve antimicrobial use at 47 US hospitals: the status of the 1997 SHEA/IDSA position paper recommendations. Infect Control Hosp Epidemiol 2000; 21:256-9.

18. Sunenshine RH, Liedtke LA, Jernigan DB, Strausbaugh LJ. Role of infectious disease consultants in management of antimicrobial use in hospitals. Clin Infect Dis 2004;38:934-8.

19. Gerding DN. The search for good antimicrobial stewardship. Jt Comm J Qual Improv 2001;27:403404.

20. Joseph J, Rodvold KA. The role of carbapenems in the treatment of severe nosocomial respiratory tract infections. Expert Opin Pharmacother 2008;9:561575. 
21. Schentag JJ, Ballow CH, Fritz AL, et al. Changes in antimicrobial agent usage resulting from interactions among clinical pharmacy, the infectious disease division, and the microbiology laboratory. Diagn Microbiol Infect Dis 1993;16:255264.

22. Scheckler WE, Bennett JV. Antibiotic usage in seven community hospitals. JAMA 1970;213:264267.

23. Society of Hospital Medicine (SHM). Definition of a Hospitalist and Hospital Medicine; November 4, 2009. Available from: http://www.hospitalmedicine.org/Web/ About_SHM/Industry/Hospital_Medicine_Hospital_Definition.aspx

24. Rohde JM, Jacobsen D, Rosenberg DJ. Role of the hospitalist in antimicrobial stewardship (ASP): a review of work completed and description of a multisite collaborative. Clin Ther 2013;35:751-7.

25. Srinivasan A. Engaging hospitalists in antimicrobial stewardship (ASP): the CDC perspective. J Hosp Med 2011;6:S31-3.

26. Yam P, Fales D, Jemison J, Gillum M, Bernstein M. Implementation of an antimicrobial stewardship program in a rural hospital. Am J Health-Syst Pharm 2012;69:1142-8.

27. Laible BR, Nazir J, Assimacopoulos AP, Schut J. Implementation of a pharmacist-led antimicrobial management team in a community teaching hospital: use of pharmacy residents and pharmacy students in a prospective audit and feedback approach. J Pharm Pract 2010; 23:531-5.

28. Davey P, Brown E, Charani E, et al. Interventions to improve antibiotic prescribing practices for hospital inpatients. Cochrane Database Syst Rev 2013;4:CD003543.

29. Vlek AL, Bonten MJ, Boel CH. Direct matrix-assisted laser desorption ionization time-of-flight mass spectrometry improves appropriateness of antibiotic treatment of bacteremia. PLoS One 2012;7:e32589.

30. Huang AM, Newton D, Kunapuli A, et al. Impact of rapid organism identification via matrix-assisted laser desorption/ionization time-offlight combined with antimicrobial stewardship team intervention in adult patients with bacteremia and candidemia. Clin Infect Dis 2013;57:1237-45.

31. Holtzman C, Whitney D, Barlam T, Miller NS. Assessment of impact of peptide nucleic acid fluorescence in situ hybridization for rapid identification of coagulase-negative staphylococci in the absence of antimicrobial stewardship intervention. J Clin Microbiol 2011;49:1581-2.

32. Forrest GN, Roghmann MC, Toombs LS, et al. Peptide nucleic acid fluorescent in situ hybridization for hospital-acquired enterococcal bacteremia: delivering earlier effective antimicrobial therapy. Antimicrob Agents Chemother 2008;52:3558-63.

33. Dodds AES, Kaye KS, DePestel DD, Hermsen ED. Antimicrobial stewardship: philosophy versus practice. Clin Infect Dis 2014;59:S112-21.

34. Barlam TF, Cosgrove SE, Abbo LM, et al. Implementing an Antibiotic Stewardship Program: Guidelines by the Infectious Diseases Society of America and the Society for Healthcare Epidemiology of America. Clin Infect Dis 2016;62:e51-77.

35. Center for Diseases Control (CDC). Core elements of hospital antibiotic stewardship programs. Atlanta, GA: US Department of Health and Human Services, CDC; 2014. Available from: http://www.cdc.gov/getsmart/healthcare/ implementation/core-elements.html

36. Schuts EC. Current evidence on hospital antimicrobial stewardship objectives: a systematic review and metaanalysis. Lancet Infect Dis 2016 [Epub ahead of print].

37. Howell EE. Antibiotic stewardship and hospitalists: the bedside is the front line. Medscape; Jun 10, 2015. Available from: http://www.medscape.com/viewarticle/845964

38. Rohde JM, Jacobsen D, Rosenberg DJ. Role of the hospitalist in antimicrobial stewardship: a review of work completed and description of a multisite collaborative. Clin Ther 2013;35:751-7. 